\title{
Mixed-Spin Kagome Strip: Classical Phase Diagram
}

\author{
S.I. Petrova ${ }^{a}$, N.B. IVANOV ${ }^{b, c, *}$ And J. SchnaCK ${ }^{c}$ \\ ${ }^{a}$ Department of Engineering Sciences and Mathematics, University of Applied Sciences, 33619 Bielefeld, Germany \\ ${ }^{b}$ Institute of Solid State Physics, Bulgarian Academy of Sciences, Tzarigradsko chaussée 72, 1784 Sofia, Bulgaria \\ ${ }^{c}$ Fakultät für Physik, Universität Bielefeld, PF 100131, D-33501, Bielefeld, Germany
}

\begin{abstract}
We analyze the classical phase diagram of a mixed-spin Heisenberg system defined on a kagome strip which is a cutout from the kagome lattice. The unit cell of the spin model contains a pair of corner-sharing triangles with four $\sigma$ spins on the outer vertices and a $S$ spin on the central site $(S>\sigma)$. The model is a natural mixed-spin extension of the previously studied spin- $\frac{1}{2}$ Heisenberg kagome strip and exhibits a rich classical phase diagram containing two collinear magnetic phases, two collinear antiferromagnetic phases with different cell spins, as well as a macroscopically degenerate canted phase occupying a region around the limit of isolated $\sigma$ chains.
\end{abstract}

DOI: 10.12693/APhysPolA.137.976

PACS/topics: spin chains, kagome strips, spin phase diagrams

\section{Introduction}

For a better understanding of the low-energy physics of the spin- $\frac{1}{2}$ Heisenberg kagome system, various modifications of the model have been studied, including different one-dimensional (1D) Heisenberg spin models defined on kagome strips [1-7]. An intriguing feature of the kagome strip models is the presence of macroscopically degenerate classical spin phases characterized by a whole branch of zero modes. Since the latter are supposed to be responsible for the special structure of the low-lying excitation spectrum of the parent kagome system, the establishment of the classical phase diagram is an important first step towards the understanding of the quantum phase diagram of the considered mixed-spin strip model. Notice that the first kagome-strip compounds $\mathrm{A}_{2} \mathrm{Cu}_{5}\left(\mathrm{TeO}_{3}\right)\left(\mathrm{SO}_{4}\right)_{3}(\mathrm{OH})_{4}(\mathrm{~A}=\mathrm{Na}, \mathrm{K})$ - built from distorted $\mathrm{CuO}_{6}$ octahedra - have already been successfully synthesized $[8,9]$, so that the study of $1 \mathrm{D}$ spin systems on kagome-strip lattices turns also into a wellmotivated intriguing task by itself.

The kagome-strip system discussed in this work (see Fig. 1) is defined by the following isotropic spin Hamiltonian:

$$
\mathcal{H}=\sum_{n=1}^{L}\left(h_{n}+J_{2}^{\prime} \sum_{\alpha=1}^{2} \boldsymbol{\sigma}_{\alpha, n} \cdot \boldsymbol{\sigma}_{\alpha+2, n+1}\right),
$$

where the cluster Hamiltonian $h_{n}$ reads

$$
h_{n}=J_{1} \sum_{\alpha=1}^{4} \boldsymbol{S}_{n} \cdot \boldsymbol{\sigma}_{\alpha, n}+J_{2}\left(\boldsymbol{\sigma}_{1, n} \cdot \boldsymbol{\sigma}_{3, n}+\boldsymbol{\sigma}_{2, n} \cdot \boldsymbol{\sigma}_{4, n}\right) \text {. }
$$

Here $S_{n}$ and $\sigma_{\alpha, n}$ are on-site quantum spin operators characterized by the quantum spin numbers $S$ and $\sigma$ $(S>\sigma)$, respectively. Index $\alpha(=1,2,3$, and 4$)$ labels

\footnotetext{
* corresponding author; e-mail: ivanov@physik.uni-bielefeld.de
}

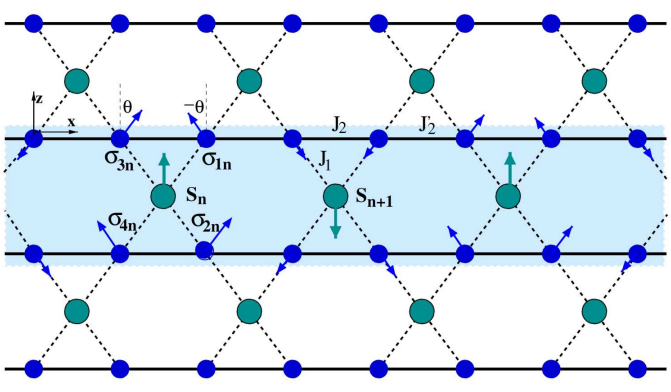

Fig. 1. Sketch of the spin strip which is selected from the kagome lattice. As an example, we also show one of the optimal classical spin configurations in the $x, z$ plane, which is constructed from the cluster ground states $C_{3}$ and $C_{4}$ shown in Fig. 2.

the four $\sigma$ spins in the unit cell, $n(=1,2, \ldots, L)$ is the cell index, and $L$ denotes the number of unit cells. Since there are two types of $\sigma-\sigma$ exchange bonds, in the general case it is reasonable to suggest $J_{2}^{\prime} \equiv \kappa J_{2} \neq J_{2}$. For simplicity, if not explicitly noticed, in what follows the considerations are restricted to the special case $J_{2}=J_{2}^{\prime}$. We use the standard parametrization $J_{1}=J \cos (t)$ and $J_{2}=J \sin (t)(0 \leq t<2 \pi)$, where the parameter $J$ serves as a measure for the energy (i.e., $J \equiv 1$ ).

\section{Classical phase diagram}

\subsection{Optimal cluster states}

The classical phase diagram of (1) can be established by using the optimal spin configurations for a separate unit cell. The latter contains a cluster of five spins: a central $S$ spin and four nearest-neighbor $\sigma$ spins (see Fig. 1). The cluster Hamiltonian $h_{n}$ exhibits two collinear (FM and FiM) and four canted $\left(C_{i}, i=1,2,3\right.$, and 4$)$ lowestenergy spin configurations (see Fig. 2) in the following intervals of the parameter $t: \pi-t_{0}<t<\frac{3 \pi}{2}(\mathrm{FM})$, $-\frac{\pi}{2}<t<t_{0}(\mathrm{FiM})$, and $t_{0}<t<\pi-t_{0}\left(C_{i}\right)$, where $i=1,2,3$ and 4 , and $t_{0}=\arctan (S / 2 \sigma)$. 

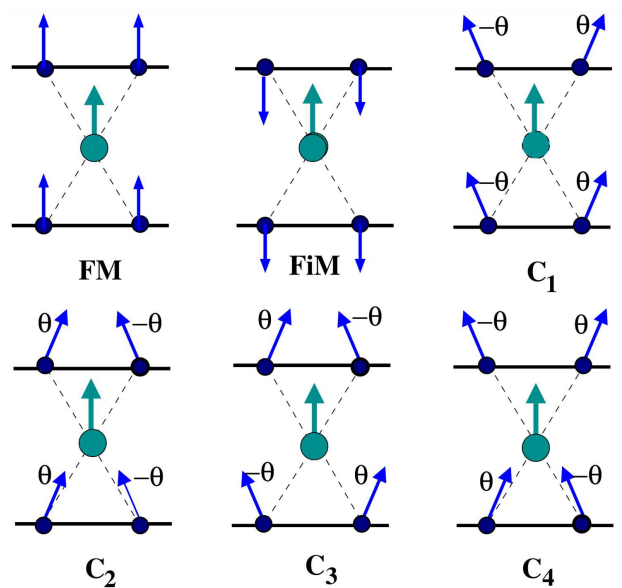

Fig. 2. The lowest-energy cluster spin configurations used as building blocks for the construction of the classical phase diagram. $\cos (\theta)=-S J_{1} / 2 \sigma J_{2}(0 \leq \theta \leq$ $\pi)$. The canted states reduce to the FM and FiM collinear states at $\pi-t_{0}$ and $t_{0}$, respectively, where $t_{0} \equiv \arctan (S / 2 \sigma)$.

\subsection{Macroscopic ground states}

Now we can use the obtained optimal cluster states as building blocks to construct the required macroscopic classical phases, by saturating the exchange $J_{2}$ bonds between the $\sigma$ spins of neighboring unit cells [10]. In practice this means that the directions of the classical $\sigma$ spins on the intercluster bonds should be exactly parallel (antiparallel) for $J_{2}<0\left(J_{2}>0\right)$. Thus, for $J_{2}<0$ the first two states in Fig. 2 can be used as building blocks to construct the fully polarized ferromagnetic (FM) and the partially polarized ferrimagnetic (FiM) phases. In the opposite case, i.e., for $J_{2}>0$, the same cluster states appear as building blocks of the collinear non-magnetic states $H_{1}$ and $H_{3}$ presented in Fig. 3a and b. The latter phases are non-degenerate, apart from trivial rotations of all spins, and are characterized by the cell spins $4 \sigma+S$ and $|4 \sigma-S|$, respectively. In the quantum version of the phase diagram, $H_{1}$ and $H_{3}$ may be expected to transform to Haldane-type (critical) non-magnetic states in the case of integer (half-integer) cell spins [11].

Turning to the parameter interval $t_{0}<t<\pi-t_{0}$, we find four GS cluster states, denoted by $C_{i}(i=1, \ldots, 4)$ in Fig. 2. Note that each of these states is degenerate with respect to rotations of a pair of nearest-neighbor $\sigma$ spins about the central $S$ spin. Below we overpass this cluster degeneracy and restrict our analysis only to coplanar spin states. As argued below, even in the subspace of coplanar configurations the classical $C$ phase in Fig. 4 is macroscopically degenerate.

To begin with, let us take the cluster configurations $C_{1}$ and $C_{2}$. In what follows we shall use the notation $C_{i}(\phi)(i=1, \ldots, 4)$ which means that the cluster spins are simultaneously rotated by the angle $\phi$ about the $y$ axis. Taking, e.g., the cluster state $C_{1}(0)$, we see that

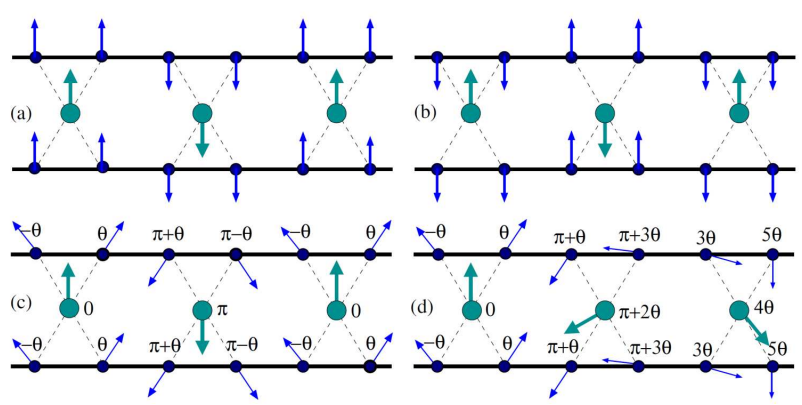

Fig. 3. Examples of macroscopic GS spin configurations built from the cluster states in Fig. 2. (a,b) The antiferromagnetic phases $H_{3}$ and $H_{1}$ with effective cell spins $4 \sigma+S$, and $|4 \sigma-S|$, respectively. (c,d) Canted and spiral GS spin configurations which can be obtained from the cluster states $C_{1}$ and $C_{2}$.

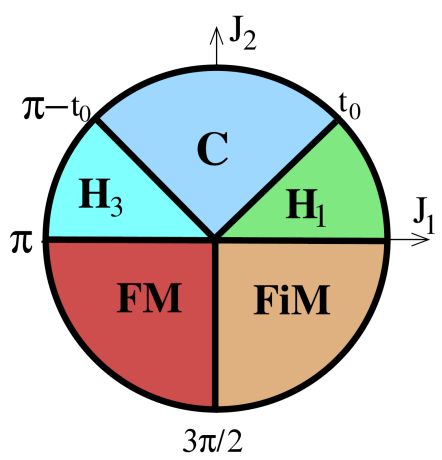

Fig. 4. Classical phase diagram of the kagome-strip model (1), containing one fully polarized (FM) and one partially polarized (FiM) collinear magnetic phases, two antiferromagnetic phases $\left(H_{1}\right.$ and $\left.H_{3}\right)$ with effective site spins $|4 \sigma \mp S|$, respectively, and a macroscopically degenerate classical spin phase $C$. The presented phase boundaries correspond to the choice $(S, \sigma)=\left(1, \frac{1}{2}\right)$ when the cell spins $|4 \sigma \mp S|$ in the phases $H_{1}$ and $H_{3}$ are 1 and 3 , respectively.

the attached cluster state can be freely rotated about the direction of the connecting $\sigma$ spins without changing the energy of the whole spin configuration. In such a way, by attaching new cluster GS's, one can construct an infinite number of optimal degenerate spin configurations [10]. As may be expected, the described degeneracy of the classical $C$ phase produces a whole branch of zero modes in the excitation spectrum. In the subspace of coplanar spin configurations, only two cluster states can be attached on the right side of $C_{1}(0)$, e.g., either $C_{1}(\pi+2 \theta)$, or $C_{2}(\pi)$. This simple rule works for any new attachment of a spin cluster and leads to the macroscopic degeneracy $2^{L}$ for a system with $L$ unit cells.

Finally, let us turn to the special coplanar spin configuration shown in Fig. 1, which is constructed from the following sequence of $C_{3}$ and $C_{4}$ cluster states $C_{3}(0) C_{4}(\pi) C_{3}(0) C_{4}(\pi) \cdots$. Interestingly, this optimal macroscopic state is the only possible one based on the $C_{3}$ and $C_{4}$ cluster states. This state is continuously 
connected to the neighboring $H_{1}$ and $H_{3}$ states in Fig. 4 at the classical phase boundaries $t=t_{0}$ and $\pi-t_{0}$, respectively. Thus, it may be speculated that this macroscopic classical state plays a special role in the quantum counterpart of the classical spin phase $C$ [11].

\section{Conclusions}

In conclusion, using Kaplan's cluster method, we have constructed the classical phase diagram of a mixed-spin Heisenberg system defined on a kagome strip. The phase diagram is characterized with a large region about the limit of independent $\sigma$ chains, which is occupied by a macroscopically degenerate spin phase. The results of this work can be used as a basis for future studies concerning the role of quantum fluctuations in the discussed Heisenberg system and, in particular, to study the properties of the quantum counterpart of the degenerate classical phase.

\section{Acknowledgments}

Support by the Bulgarian Science Foundation grant DN0818/14.12.2016 is gratefully acknowledged.

\section{References}

[1] P. Azaria, C. Hooley, P. Lecheminant, C. Lhuillier, A.M. Tsvelik, Phys. Rev. Lett. 81, 1694 (1998)

[2] S.K. Pati, R.R.P. Singh, Phys. Rev. B 60, 7695 (1999).

[3] S.R. White, R.R.P. Singh, Phys. Rev. Lett. 85, 3330 (2000).

[4] A. Donkov, A.V. Chubukov, Eur. Phys. Lett. 80 , 67005 (2007).

[5] T. Shimokawa, H. Nakano, J. Phys. Soc. Jpn. 81, 084710 (2012).

[6] K. Morita, T. Sugimoto, Sh. Sota, T. Tohyama, Phys. Rev. B 97, 014420 (2018).

[7] A.A. Donkov, N. B. Ivanov, J. Richter, AIP Conf. Proc. 2075, 020015 (2019).

[8] Y. Tang, W. Guo, H. Xiang, S. Zhang, M. Yang, M. Cui, N. Wang, Zh. He, Inorg. Chem. 55, 644 (2016).

[9] W. Guo, Y. Tang, J. Wang, Zh. He, Inorg. Chem. 56, 1830 (2017).

[10] T.A. Kaplan, Phys. Rev. B 80, 012407 (2009).

[11] N.B. Ivanov, J. Schnack, J. Richter, to be published. 